\title{
Mathematical Modeling of the Dynamics of
}

\section{Air Jet Crossing the Canopy of Tree Crops during}

\section{Pesticide Application}

\author{
Dario Friso* \\ Department of Land, Environment, Agriculture and Forestry - TESAF \\ Research Unit: Agricultural and Food Engineering \\ University of Padova, Viale dell'Università 16, Legnaro 35020, Padova, Italy \\ *Corresponding author \\ Cristiano Baldoin \\ Department of Land, Environment, Agriculture and Forestry - TESAF \\ Research Unit: Agricultural and Food Engineering \\ University of Padova, Viale dell’Università 16, Legnaro 35020, Padova, Italy \\ Fabio Pezzi \\ Department of Agricultural and Food Sciences \\ University of Bologna, Italy \\ Copyright (C2015 Dario Friso, Cristiano Baldoin and Fabio Pezzi. This is an open access \\ article distributed under the Creative Commons Attribution License, which permits unrestricted \\ use, distribution, and reproduction in any medium, provided the original work is properly cited.
}

\begin{abstract}
A mathematical model was developed to get an equation of the decrease of air velocity crossing the canopy of tree crops during pesticide application using air carrier orchard sprayers. The utility of such a model rises from the need for an aid to understand the experimental results of several authors, who agree with the opinion that air jet velocity greatly affects environmental pollution from pesticides. Further, probably in the future it will arise the demand to implement the equation of air velocity decay in self-adjustment systems of the fan installed on orchard sprayers to limit spray drift. Based on momentum theorem applied under three assumptions, a differential equation was found and its integration lead to a closed
\end{abstract}


solution that can easily be implemented in a PLC for the self-adjustment system to develop. The integral equation thus obtained, together with the assumptions made, was submitted to on-field verification on three crops (peach, vine and apple). The results show a good correspondence between measured and estimated air speed as predicted by the mathematical model, with a relative mean error $3.3 \%$ and a maximum value of $6.2 \%$.

Keywords: Mathematical modeling; Momentum theorem; Velocity decay equation; Air jet; Air-assisted sprayer; Orchard; Vineyard; Agricultural engineering

\section{Introduction}

Air assisted sprayers are widely used for spray application in fruit and vine growing. The air jet is often produced by an axial fan, less frequently by a centrifugal one; anyway, the air stream first hits the nozzles, then drags the droplets to cross the crop row.

It is easy to understand that air velocity of the jet strongly affect the effectiveness of treatment, with particular reference to the fraction of spray that the canopy can retain compared to the total sprayed amount. Since the amount not retained is lost in the environment, it is necessary to reduce this fraction, not only for economic reasons, but also for ecological issues.

For example, a recent research showed that in peach orchards with given dimensions, the velocity of the air jet entering the canopy affects spray losses [1]; in particular, when velocity is too high, the likelihood of alignment of leaves according to air direction increases, thus causing drifting of a relevant percentage of droplets beyond the tree row.

The results of further specific experiments in vineyard [2] indirectly confirm this assumption. By the way, thirty years ago [3], based on experiments carried out in Hungary, it was indicated that a velocity of the air jet over $2 \div 3 \mathrm{~m} / \mathrm{s}$ (referred as the greater value along the cross profile) at the exit of the tree row causes a decrease of droplets retention in the canopy, hence increasing drift.

Therefore, it seems to be useful, aiming to a better understanding of experimental results, to have available an equation of the velocity of the air jet as a function of the distance from the outlet of the sprayer fan, as air crosses the canopy.

It could also be useful to provide the sprayers with a self-adjusting system to set the fan speed to keep the optimal air jet velocity at the exit of the canopy, both respect to the height of the row [1] and to the growth stage [4].

This equation is well known with reference to a jet freely diffusing in the atmosphere [5]. It is useful to link the velocity of air entering the row with the one at the fan outlet; this last one is directly proportional to fan flow rate and hence to fan speed. To complete the data, it is necessary to find the equation of the air velocity crossing the tree row.

The aim of this paper is to find a solution to the mathematical problem of the velocity decay of the air jet as it crosses the foliage layers of the tree row. It must 
be a closed solution, as made in previous researches [6,7], having the same objective to obtain an equation to be easily implemented in a control system. In this case in the hypothesized [4] possible self-adjustment system for orchard sprayers.

The equation of air velocity decrease along the tree foliage lies upon three assumptions:

1. foliage is uniformly distributed in space, and subsequently the LAD (Leaf Area Density) is constant;

2. forward motion of the sprayer is ignored, since its velocity is one order of magnitude lower than air velocity from fan exit, and no effect of travel speed on spray deposition in the canopy was experimentally observed [8];

3. inverse relationship between drag coefficient and air velocity is considered.

The third assumption, also proposed by other authors $[9,10]$, has been following verified with a wind tunnel tests.

Finally, the obtained equation was confirmed by a field test, measuring the velocity of the air out of the row compared to the one entering on three crops: vine, peach and apple.

\section{Velocity of air jet free diffusing in the atmosphere}

A short review of the theory of an air jet freely diffusing in the atmosphere, adapted to the sprayers [5], is useful to introduce the mathematical development of the axial velocity decay equation of air jet crossing the canopy of tree crops. According to Brazee [5], an air jet diffusing in the atmosphere and exiting from an arc-shaped outlet (as in most sprayers) $2 b_{o}$ wide, is marked out for three zones (fig. 1): the first one, close to the outlet (initial region), the second (transitional region) and the third (main region).

Since in the output section air velocity $v_{x o}$ is constant along the whole section (irrotational flow), the initial region includes a wedge-shaped core where air velocity remains constant and equal to $v_{x o}$, and a peripheral part where diffusion with ambient air starts, thus including this last one within the jet.

In the transitional region, diffusion goes ahead extending also to the central section, where the axial velocity $v_{x}$, which is also the maximum velocity on the transverse profile of the jet, progressively decays as distance from the outlet increases.

In the third region, the diffusion is fully established, so the diffusion triangle is open at most; its sides limit, for this region, the motion field from the calm air. 


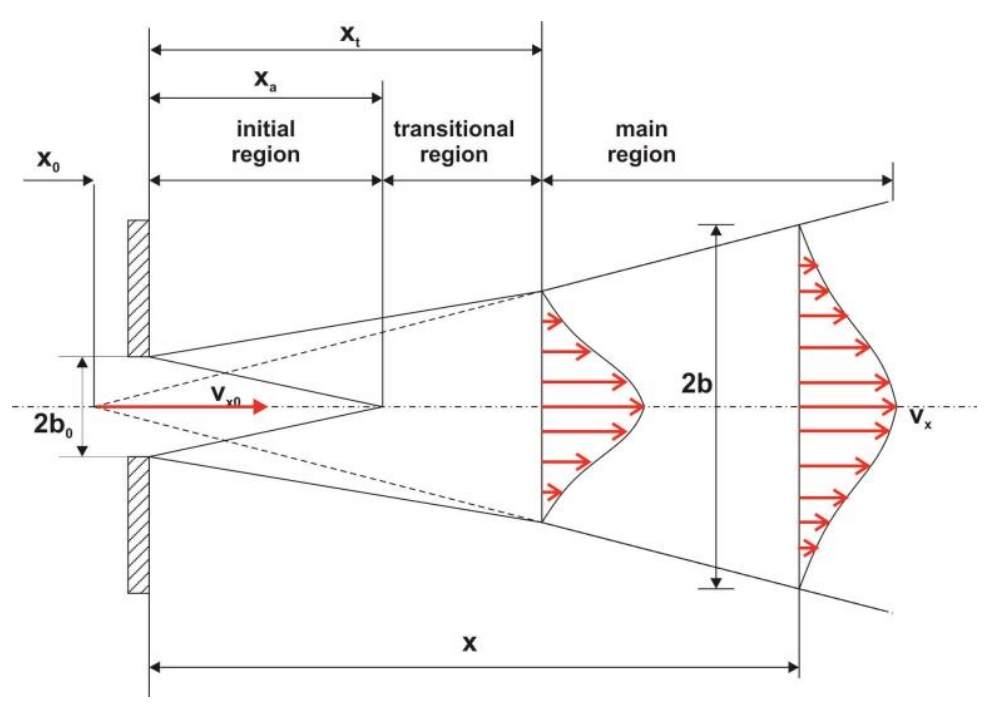

Fig. 1 - Diagram of an ideal jet in free diffusion

In the third main region, the profiles of velocity are characterized by similitude; Schlighting [11] found that the integral mean velocity on the width $2 b, v_{x m}$, is a fraction $A_{1}=0,316$ of the maximum velocity (on centerline) $v_{x}$, with $A_{l}$ constant and independent from the distance $x$. Also the integral mean of the squared velocity on the width $2 b, v_{x m}^{2}$ is a fraction $A_{2}=0,45$ of the maximum squared velocity (on centerline) $v_{x}^{2}$, with $A_{2}$ constant and independent from the distance $x$ :

$$
\begin{aligned}
& v_{x m}=A_{1} \cdot v_{x} \\
& v_{x m}^{2}=A_{2} \cdot v_{x}^{2}
\end{aligned}
$$

Considering these relationships and under the assumptions of absence of wind and null forward velocity of air-assisted sprayer, Fox et al. [12] and Randall [13] proposed that maximum axial air velocity $v_{x}$, i.e. in centerline, decreases as $x$ increases according to the following equation, obtained through the momentum conservation law:

$$
v_{x}=3.8\left(\frac{n_{2} \cdot b_{o} \cdot r_{o}}{\left(x-x_{o}\right)\left(x+r_{o}\right)}\right)^{0.5} \cdot v_{x o}
$$

where (see figs. 1 e 2$): v_{x o}$ is the maximum velocity at sprayer output $(\mathrm{m} / \mathrm{s}) ; 2 b_{o}$ is the width of the outlet section $(\mathrm{m}) ; x$ is the distance travelled by the air jet from the outlet $(\mathrm{m}) ; x_{o}$ is the distance, normally negative, between the vertex of the diffusion triangle and the outlet section $(\mathrm{m}) ; r_{o}$ is the radius of the border of air outlet from the sprayer (vertical view of fig. 2); $n_{2}$ is the ratio between the integral mean of the squared velocity on the width $2 b_{o}$ (fig. 1 ) of the outlet section and the maximum squared velocity (on centerline) in the same outlet section. 
Rather than assuming the irrotational motion in the outlet section, and therefore $n_{2}=1$, Brazee [5] assumed $n_{2}=0.76$. This allowed him to ignore the transition zone, because he had experimentally found that this zone was very small. Therefore, it is possible to consider a direct pass between the initial region and the main region $\left(x_{a}=x_{t}\right)$, with the advantage that distance $x_{o}$ can be more easily calculated with a good approximation according to:

$$
\begin{gathered}
x_{o}=x_{t}-\frac{n_{2} \cdot b_{o} \cdot r_{o}}{C_{m} \cdot A_{2} \cdot\left(x_{t}+r_{o}\right)} \\
x_{t}=x_{a}=-\frac{r_{o}}{2}+\left(\frac{r_{o}^{2}}{4}+\frac{b_{o} \cdot r_{o}}{0.27 \cdot\left(1-2 A_{1}+A_{2}\right)}\right)^{0.5}
\end{gathered}
$$

where: $x_{a}$ e $x_{t}$ are understandable from fig. $1 ; C_{m}$ is the tangent of the vertex semi-angle of the diffusion triangle (figs. 1 e 2) and its value is $0,22[5,13]$; the diffusion triangle represents the shape of the air jet that proceeds diffusing with semi-width $b$ :

$$
b=C_{m} \cdot\left(x-x_{o}\right)
$$

\section{Velocity of the air jet in the canopy}

\subsection{First and second assumptions}

Figure 2 shows the section of a tree row with the air jet coming from the sprayer. Let us consider the assumption that foliage is uniform and hence the jet crosses it keeping its width $2 b$ according to the equation (6). We know that this assumption is not actually verified, both because leaves are not evenly distributed in the foliage and because of the various sized branches in the canopy. Nevertheless, it is necessary to the mathematical development, and the final equation includes a parameter depending on the features of the foliage as well: the experimental determination of this parameter will automatically correct the equation. This method is very frequent in engineering sciences.

As mentioned in the introduction, the second assumption refers to the analysis of the air jet dynamics without considering the forward motion of the sprayer. This is acceptable because the working speed of the sprayers $v_{f}$ is normally between 2 and $4 \mathrm{~m} / \mathrm{s}$, about one order of magnitude lower than the velocity of the air jet $v_{x o}$, which ranges between 20 and $30 \mathrm{~m} / \mathrm{s}$.

\subsection{The control volume}

Fig. 2 shows in particular a control volume for the application of the momentum theorem to get the equation of air velocity $v_{x}(x)$ in the foliage as a function of the distance $x$. The control volume, on the horizontal plane at distance $x$ from the outlet 
section of the sprayer, is $2 b=2 C_{m} \cdot\left(x-x_{o}\right)$ wide and has an infinitesimal thickness equal to $d x$. On the vertical plane, the control volume appears as a sector of an annulus, with thickness $d x$, subtending an angle $\beta$ and with the inner arc equal to $\beta \cdot\left(r_{o}+x\right)$, where $r_{o}$ is the radius of the outlet border of the sprayer.

So the control volume results:

$$
d V=2 C_{m} \cdot \beta \cdot\left(r_{o}+x\right) \cdot\left(x-x_{o}\right) \cdot d x
$$

while the inlet and outlet areas are:

$$
\begin{gathered}
a=2 C_{m} \cdot \beta \cdot\left(r_{o}+x\right) \cdot\left(x-x_{o}\right) \\
a+d a=2 C_{m} \cdot \beta \cdot\left(r_{o}+x+d x\right) \cdot\left(x+d x-x_{o}\right)
\end{gathered}
$$

After having specified the leaf area density $\rho_{l}$, also known as $L A D\left(\mathrm{~m}^{2} / \mathrm{m}^{3}\right)$, as the ratio between the area of the leaves included in a given volume, and after having considered it as constant due to the assumption of the uniformity of the foliage (see par. 3.1), we can write the leaf area $d S$ within the control volume as follows:

$$
d S=q \cdot d V=q \cdot 2 C_{m} \cdot \beta \cdot\left(r_{o}+x\right) \cdot\left(x-x_{o}\right) \cdot d x
$$
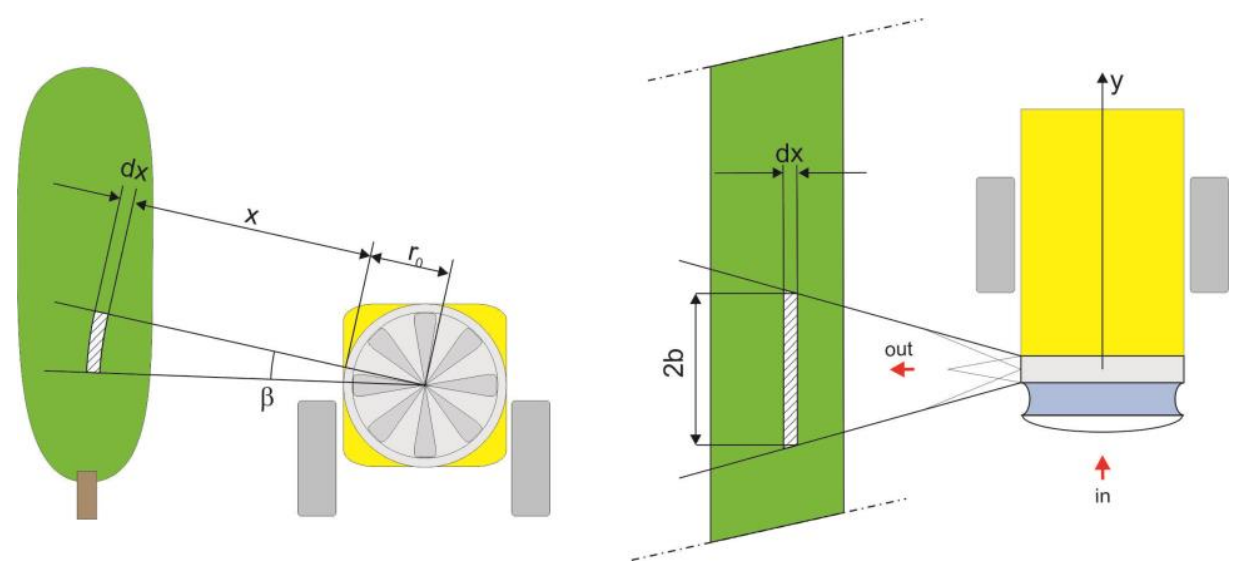

Fig. 2 - Geometrical elements defining the control volume: vertical view (left), plan view (right).

\subsection{Momentum theorem}

The application of the momentum theorem [14] to the control volume [15] establishes that the difference between the ingoing momentum flux and outgoing momentum flux shall equal the drag force opposed by the foliage items (leaves and branches), considered according to the assumption of par. 3.1 as uniformly distributed within the control volume.

The ingoing momentum flux, also according to (6), results: 


$$
p_{i}=\rho_{a} \cdot \beta \cdot\left(r_{o}+x\right) \cdot \int_{2 b} v^{2} \cdot d y=\rho_{a} \cdot \beta \cdot\left(r_{o}+x\right) \cdot 2 C_{m} \cdot\left(x-x_{o}\right) \cdot v_{x m}^{2}
$$

where: $\rho_{a}$ is the air density; $v_{x m}^{2}=\frac{1}{2 C_{m}\left(x-x_{o}\right)} \int_{2 b} v^{2} \cdot d y$ is the integral mean of the squared values of velocities along the width $2 b$. We can also write that $v_{x m}^{2}=A_{2}^{\prime} \cdot v_{x}^{2}$, where $v_{x}$ is the maximum velocity respect to the width $2 b$ and $A_{2}^{\prime}$ is unknown, because it depends on the way the foliage interacts with air. Therefore, it may also differ from the $A_{2}$ in equation (2), but, as well as $A_{2}$, also $A^{\prime}{ }_{2}$ can be considered as constant related to $x$ because the canopy is assumed as uniform along $x$ axis too, according to the assumption in par. 3.1.

Finally, momentum flux ingoing $p_{i}$ and outgoing $p_{e}$ are respectively:

$$
\begin{gathered}
p_{i}=\rho_{a} \cdot \beta \cdot\left(r_{o}+x\right) \cdot 2 C_{m} \cdot\left(x-x_{o}\right) \cdot A_{2}^{\prime} \cdot v_{x}^{2} \\
p_{e}=\rho_{a} \cdot \beta \cdot\left(r_{o}+x+d x\right) \cdot 2 C_{m} \cdot\left(x+d x-x_{o}\right) \cdot A_{2}{ }_{2} \cdot\left(v_{x}+d v_{x}\right)^{2}
\end{gathered}
$$

Their difference, after development and ignoring the higher order infinitesimals, results:

$$
p_{e}-p_{i}=\rho_{a} \cdot 2 C_{m} \cdot \beta \cdot A_{2}^{\prime}\left[\left(2 x-x_{o}+r_{o}\right) v_{x}^{2}+\left(x-x_{o}\right)\left(x+r_{o}\right) 2 \cdot v_{x} \frac{d v_{x}}{d x}\right] d x
$$

Inside the control volume, the resulting drag force is:

$$
d T=-\frac{1}{2} \rho_{a} \cdot c_{r} \cdot d S \cdot v_{x m}^{2}=-\frac{1}{2} \rho_{a} \cdot c_{r} \cdot g \cdot 2 C_{m} \cdot \beta \cdot\left(r_{o}+x\right) \cdot\left(x-x_{o}\right) \cdot A_{2}^{\prime} \cdot v_{x}^{2} \cdot d x
$$

where: the drag force $d T$ is opposite to velocity $v_{x} ; c_{r}=c_{r}\left(v_{x}\right)$ is the unknown adimensional drag coefficient and $\rho_{l}\left(\mathrm{~m}^{2} / \mathrm{m}^{3}\right)$ is leaf area density. Making (14) and (15) equal, with respect of momentum theorem and simplifying, it results a ODE:

$$
\frac{d v_{x}}{d x}+\frac{\left(2 x-x_{o}+r_{o}\right)}{2\left(x-x_{o}\right)\left(x+r_{o}\right)} v_{x}+\frac{1}{4} \rho_{l} \cdot c_{r} \cdot v_{x}=0
$$

\subsection{Third assumption}

Fraser [9] showed by wind tunnel testing that drag coefficient $c_{r}$ of the foliage of conifer trees is inversely proportional to air velocity when this last have high values enough. Further, Tsatsarelis [10] observed that this is verified in broad-leaved trees when air velocity $v_{x}$ exceeds $3 \mathrm{~m} / \mathrm{s}$ and deduced that, beyond this limit, the leaves begin to direct themselves increasingly according to the direction of the airstream as result of streamlining, thus leading to a higher decay of the drag coefficient. This last one becomes $c_{r}=\frac{k}{v_{x}}$, respect to the classic law 
such as $c_{r}=\frac{k^{\prime}}{R e^{n}}$ where $R e$ is Reynolds number and $n<1$ related to the transitional motion, or $n=0$ related to the turbulent motion along fixed objects. The differential equation (16), with $v_{x} \geq 3$ becomes then a linear ODE:

$$
\frac{d v_{x}}{d x}+\frac{\left(2 x-x_{o}+r_{o}\right)}{2\left(x-x_{o}\right)\left(x+r_{o}\right)} v_{x}+\frac{1}{4} k \cdot \rho_{l}=0
$$

\subsection{Solution of the linear differential equation}

Solving the (17) it results:

$$
v_{x}=\frac{C}{G(x)}-\frac{k \cdot \rho}{16}\left[\left(2 x-x_{o}+r_{o}\right)-\frac{\left(r_{o}+x_{o}\right)^{2}}{G(x)} \ln \left(\sqrt{r_{o}+x}+\sqrt{x-x_{o}}\right)\right]
$$

where: $G(x)=\sqrt{\left(x-x_{o}\right) \cdot\left(x+r_{o}\right)} ; C$ is the constant of integration, obtainable by imposing the conditions $v_{x}=v_{x i}$ for $x=x_{i} ; x_{i}$ and $v_{x i}$ are respectively the coordinate and the velocity of air entering the foliage; this last is known by applying the (3) for $x=x_{i}$ :

$$
v_{x_{i}}=3.8\left(\frac{n_{2} \cdot b_{o} \cdot r_{o}}{\left(x_{i}-x_{o}\right)\left(x_{i}+r_{o}\right)}\right)^{0.5} \cdot v_{x o}
$$

Therefore, the (18) becomes:

$$
v_{x}=v_{x i} \frac{G\left(x_{i}\right)}{G(x)}-\frac{k \cdot \rho_{i}}{16}\left[\left(2 x-x_{o}+r_{o}\right)-\left(2 x_{i}-x_{o}+r_{o}\right) \frac{G\left(x_{i}\right)}{G(x)}-\frac{\left(r_{o}+x_{o}\right)^{2}}{G(x)} \ln \left(\frac{\sqrt{r_{o}+x}+\sqrt{x-x_{o}}}{\sqrt{r_{o}+x_{i}}+\sqrt{x_{i}-x_{o}}}\right)\right]
$$

With the typical values of $x, x_{i}, r_{o}, x_{o}$ in orchard and vineyard spraying, the contribution of the third addend into the square brackets is negligible, therefore:

$$
v_{x}=v_{x i} \frac{G\left(x_{i}\right)}{G(x)}-\frac{k \cdot p}{16}\left[\left(2 x-x_{o}+r_{o}\right)-\left(2 x_{i}-x_{o}+r_{o}\right) \frac{G\left(x_{i}\right)}{G(x)}\right]
$$

The constant $C$ can also be calculated applying the conditions at the exit of the foliage: $v_{x}=v_{x e}\left(\right.$ for example, according to Trefan [3]: $\left.v_{x}=v_{x e}=3 m / s\right)$ for $x=x_{e}$. Therefore, the (21) becomes:

$$
v_{x}=v_{x e} \frac{G\left(x_{e}\right)}{G(x)}-\frac{k \cdot p_{l}}{16}\left[\left(2 x-x_{o}+r_{o}\right)-\left(2 x_{e}-x_{o}+r_{o}\right) \frac{G\left(x_{e}\right)}{G(x)}\right]
$$


Dividing the (3) by the (19), it results:

$$
v_{x}=v_{x i} \frac{G\left(x_{i}\right)}{G(x)}
$$

So the (21) is just the extension of the (23), that means of the (3), when the air crosses a volume with foliage $(p>0)$. It should be reminded that the (21) and (23) are valid under the assumption that velocity is $v_{x} \geq 3$ thus resulting: $c_{r}=\frac{k}{v_{x}}$. Further, they are also valid when foliage is uniform (par. 3.1), and therefore $\rho_{l} \mathrm{e}$ $A^{\prime} 2$ are constant respect to $x$. The factor $q \cdot k$ is to be determined experimentally, thus correcting also the simplification coming from the assumption of the uniformity of foliage in par. 3.1.

\section{Experimental evaluation}

The theoretical development of the equations (21) and (22) included the assumption (par. 3.4), according to Tsatsarelis [10], that for $v_{x} \geq 3$, the relationship between the drag coefficient $c_{r}$ and the velocity $v_{x}$ is like: $c_{r}=\frac{k}{v_{x}}$. Consequently, the experimental focused first on the verification of this assumption. Second, a field test was carried out to measure the velocity of air on exit from the rows of three tree crops, then comparing them with the values predicted by the equation (21).

\subsection{Materials and methods}

To verify the assumption in par.3.4, represented by the $c_{r}=\frac{k}{v_{x}}$, a wind tunnel was built, made up of a square section $0.8 \times 0.8 \mathrm{~m}^{2}$ and $5 \mathrm{~m}$ long tunnel. A twin centrifugal fan was installed on one end, with a maximum flow rate of $7 \mathrm{~m}^{3} / \mathrm{s}$ and air velocity up to $11 \mathrm{~m} / \mathrm{s}$. A variable speed gearbox between the electric motor and the fan, together with the possibility to reduce the intake area, allowed adjusting the air velocity $v_{x m}$ step by step from 0.5 up to $11 \mathrm{~m} / \mathrm{s}$. The tunnel was equipped with a honeycomb flow straightener, after which the air speed meter was placed. The probe vane $(10 \mathrm{~mm}$ diameter) was connected to a data logger and it was calibrated to record each test as a mean of 64 values, each one measured shifting the placement of the probe along the tunnel section. The air jet hit a plant of Ficus beniamina L. Placed before the exit of the tunnel; the trunk of the plant stuck out from the base through a slot, so that the pot containing the roots was connected to a dynamometer to measure the force $T(\mathrm{~N})$.

After the test, the leaf area density $\rho_{l}\left(\mathrm{~m}^{2} / \mathrm{m}^{3}\right)$ of the plant was measured, taking out le leaves included in a known volume $V$.

This way the trend of the quantity $k=c_{r} \cdot v_{x m}$ as affected by air velocity $v_{x m}$, was assessed, according to: 


$$
|T|=\frac{1}{2} c_{r} \cdot \rho_{a} \cdot V \cdot \rho_{l} \cdot v_{x m}^{2} \Rightarrow k=c_{r} \cdot v_{x m}=\frac{2 \cdot|T|}{\rho_{a} \cdot V \cdot \rho_{l} \cdot v_{x m}}
$$

thus verifying in which range of air velocity the mentioned quantity $k$ resulted constant and, therefore, the inverse proportionality relationship $c_{r}=\frac{k}{v_{x}}$ was applicable.

The same vane probe and data logger were also used to measure and record the air velocity $v_{x e}$ at the exit from the tree row of the three crops (vine, peach and apple) in order to verify experimentally the equation (21). Each of the found values is the mean of 5 replications obtained by moving forward the sprayer 1 meter each time along the inter-row. Each replication considered the measurement at a constant height from the ground, equal to 1 meter.

For each of the three crops, 3 tests were performed, with 3 different air velocity $v_{x o}$ at the outlet of the sprayer $(20.9,24.5$ and $28.7 \mathrm{~m} / \mathrm{s})$ and therefore 3 different velocities of air $v_{x i}$ entering the foliage (table 1).

Considering the inter-row distances and the different widths of the rows of the three crops, the values (means out of 5 measurements, 1 meter constant distance from the ground) of the horizontal coordinates $x_{i}$ e $x_{e}$ ingoing and outgoing the row are reported in table 1.

With reference to figures 1 and 2, table 1 shows also the values of the quantities $r_{o}$ and $b_{o}$, measured on the sprayer, and $x_{t}$ and $x_{o}$, calculated with the equations (4) and (5).

For each crop, the mean values of the quantities $r_{o}, x_{o}, x_{i}, x_{e}, v_{x i}$ and $v_{x e}$ (tab. 1) and of the derived ones $G\left(x_{i}\right)=\sqrt{\left(x_{i}-x_{o}\right) \cdot\left(x_{i}+r_{o}\right)}, G\left(x_{e}\right)=\sqrt{\left(x_{e}-x_{o}\right) \cdot\left(x_{e}+r_{o}\right)}$, were used to find the unknown variable, here named resistance factor, $k \cdot p_{l}$ by means of the equation (21):

$$
k \cdot q=16\left(v_{x i} \frac{G\left(x_{i}\right)}{G\left(x_{e}\right)}-v_{x e}\right) \cdot\left[\left(2 x_{e}-x_{o}+r_{o}\right)-\left(2 x_{i}-x_{o}+r_{o}\right) \frac{G\left(x_{i}\right)}{G\left(x_{e}\right)}\right]^{-1}
$$

\section{Results and discussion}

The tests in wind tunnel carried out to confirm the assumption made in par. 3.4, described by the $c_{r}=\frac{k}{v_{x}}$, after having applied the equation (24), allowed to calculate the values of $k$ as shown in the diagram of fig. 3. $k$ results nearly constant and equal to a mean value of $1.1 \mathrm{~m} / \mathrm{s}$ since an air velocity in tunnel $v_{x m}=4 \mathrm{~m} / \mathrm{s}$. Anyway, with an error $<5 \%, k$ can be assumed as invariant up to the air velocity of $3 \mathrm{~m} / \mathrm{s}$, thus substantially confirming the results found by Tsatsarelis [10].

The value of leaf area density $\rho_{l}$, essential to apply the (24), resulted $4.5 \mathrm{~m}^{2} / \mathrm{m}^{3}$. 


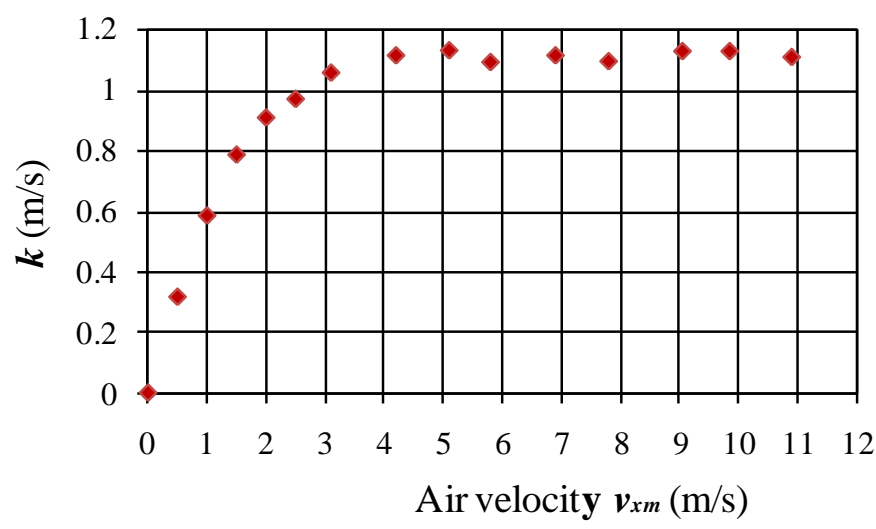

Fig. 3 - Relationship between $k$ parameter and air velocity $v_{x m}$.

Table 1 reports the results of the field test as referred to the mean values of maximum air velocity $v_{x e}$ out of the tree row and the relative standard deviation (S.D.). These last ones resulted higher in peach orchard, because of the greater non uniformity of the foliage compared to vine and apple.

For each crop, table 1 reports also the resistance factor $k \cdot \rho_{l}$ obtained from equation (25) considering the mean values of $v_{x i}$ and $v_{x e}$. Then the equation (21) was calculated using the resistance factors $k \cdot \rho_{l}$ to get the three values of air velocity on exit $v_{x e}$-equation (21) - corresponding to the respective values of $v_{x i}$.

Using equation (3) (table 1), it was also calculated the velocity of the free diffusing air jet $v_{x e}$, namely like in absence of the tree row, at a distance $x_{e}$.

For a complete presentation of the results, figures 4 and 5 show the trends of maximum air velocities $v_{x}$ relative to the horizontal distance $x$ from the outlet of the sprayer. The dotted curves, obtained with the equation (3), refer to the free jet and show that maximum air velocity (on centerline) is constant and equal to $v_{x o}$ in the initial region up to $x_{t}=x_{a}$.

The diagrams, from distance $x_{i}$ also show the curves of decay of velocity $v_{x}$ within the canopy as predicted by the equation (21). Finally, the experimental values of $v_{x e}$ and the relative standard deviation bars are shown.

The graphs highlight that the decay of air velocity in the foliage, compared to the free jet, is greater in vine and apple and smaller in peach, according to the minor resistance factor $k \cdot \rho_{l}$ of this last crop.

Table 1 - Geometrical data measured on the crops and on the sprayer and air velocities measured and predicted.

\begin{tabular}{|c|c|c|c|}
\hline Crop & Peach & Vines & Apple \\
\hline Training system & Open center & Cane & Central leader \\
\hline$r_{o}(\mathrm{~m})$ & & 0.47 & \\
\hline$b_{o}(\mathrm{~m})$ & & 0.075 \\
\hline$x_{o}(\mathrm{~m})$ & & -0.085 \\
\hline$x_{t}(\mathrm{~m})$ & 0.373 & \\
\hline
\end{tabular}


Table 1 (Continued): - Geometrical data measured on the crops and on the sprayer and air velocities measured and predicted.

\begin{tabular}{|c|c|c|c|c|c|c|c|c|c|}
\hline$x_{i}(\mathrm{~m})$ & \multicolumn{3}{|c|}{0.67} & \multicolumn{3}{c|}{0.71} & \multicolumn{3}{c|}{0.63} \\
\hline$x_{e}(\mathrm{~m})$ & \multicolumn{3}{|c|}{2.32} & \multicolumn{3}{c|}{1.94} & \multicolumn{3}{c|}{} \\
\hline$v_{x o}(\mathrm{~m} / \mathrm{s})$ & 20.9 & 24.5 & 28.7 & 20.9 & 24.5 & 28.7 & 20.9 & 24.5 & 28.7 \\
\hline$v_{x i}(\mathrm{~m} / \mathrm{s})$ & 14.0 & 16.4 & 19.2 & 13.4 & 15.7 & 18.4 & 14.7 & 17.2 & 20.1 \\
\hline$v_{x e}(\mathrm{~m} / \mathrm{s})$ experim. & 3.5 & 4.2 & 5.6 & 3.1 & 4.0 & 5.6 & 2.7 & 3.9 & 5.1 \\
\hline S.D. of $v_{x e}$ & 0.9 & 0.8 & 0.5 & 0.6 & 0.5 & 0.6 & 0.2 & 0.4 & 0.5 \\
\hline$k \cdot \rho_{l}\left(\mathrm{~s}^{-1}\right)$ & \multicolumn{3}{|c|}{5.3} & \multicolumn{3}{|c|}{12.2} & \multicolumn{3}{c|}{10.4} \\
\hline$v_{x e}(\mathrm{~m} / \mathrm{s})$ eq. $(21)$ & 3.5 & 4.4 & 5.4 & 3.2 & 4.2 & 5.4 & 2.9 & 3.9 & 5.0 \\
\hline$v_{x e}(\mathrm{~m} / \mathrm{s})$ eq. $(3)$ & 5.0 & 5.9 & 6.9 & 5.9 & 6.9 & 8.1 & 5.5 & 6.5 & 7.6 \\
\hline
\end{tabular}
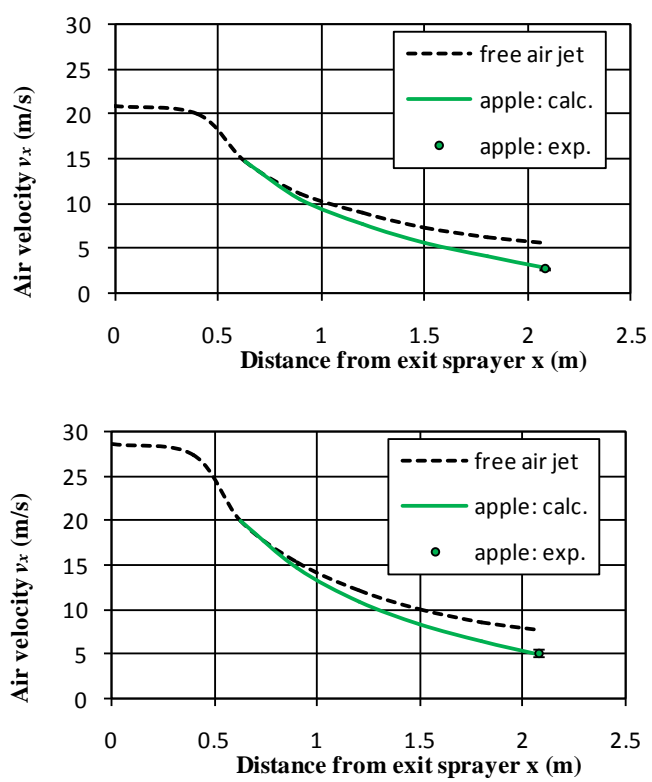

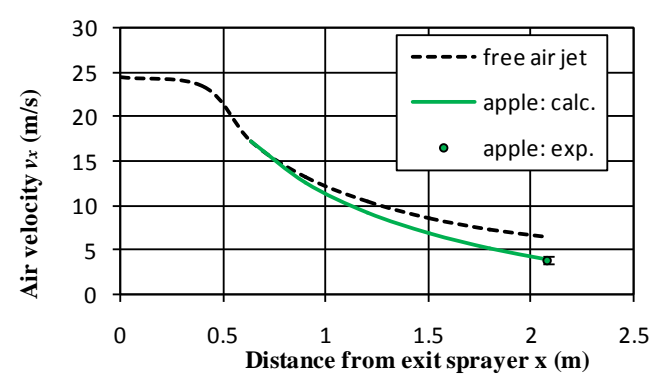

Fig. 4 - Maximum axial velocity $v_{x}$ of free air jet (centerline) related to distance $x$ (- - - -), predicted by eq. (3). The maximum velocity of air jet in the foliage, predicted by eq. (21) is also shown for apple trees (-----), with the experimental value $\left({ }^{\circ}\right)$ at distance $x_{e}$. Graphs are sorted clockwise for increasing air velocity on exit from the sprayer $v_{x o}$. 

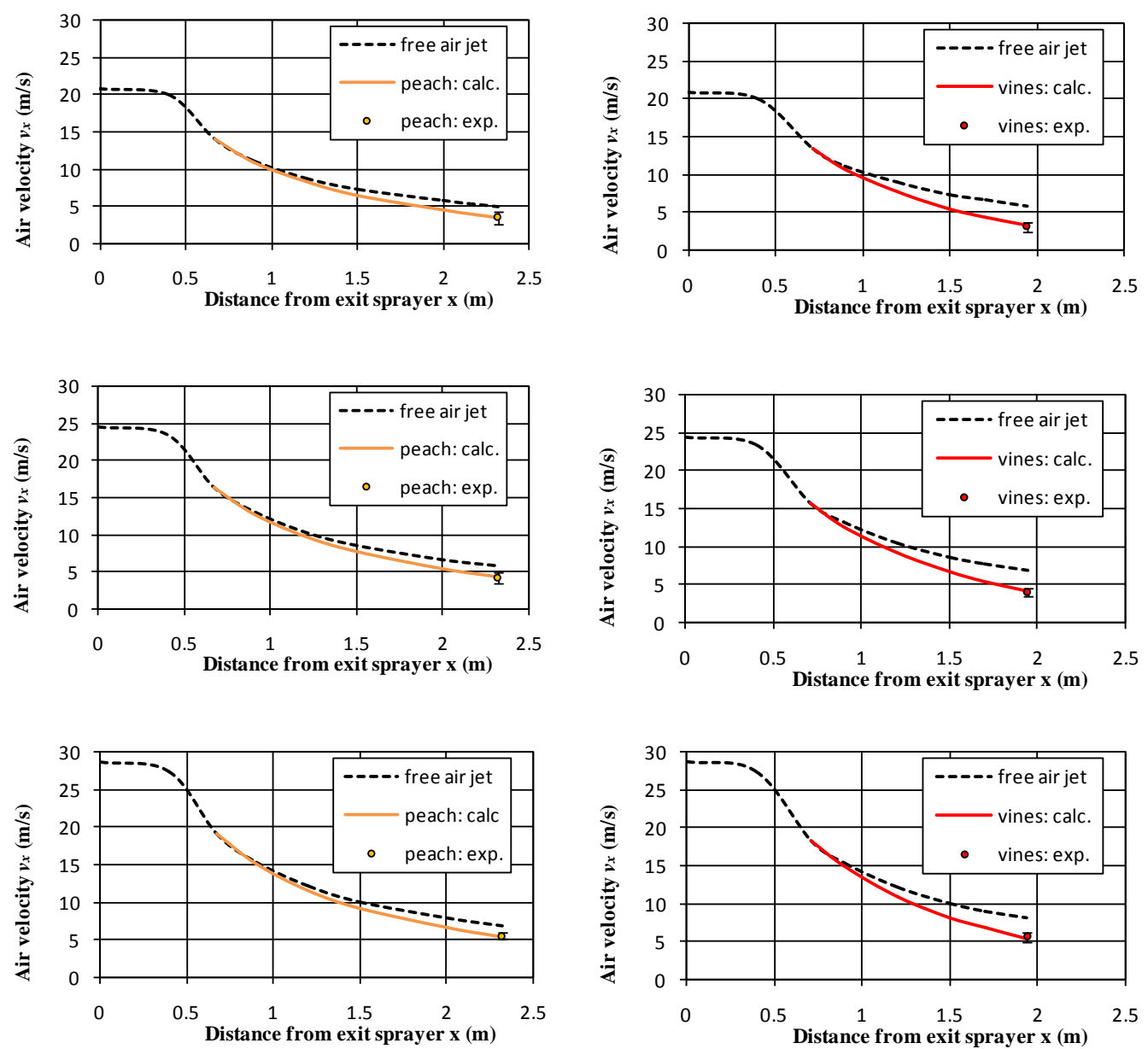

Fig. 5 - Maximum axial velocity $v_{x}$ of free air jet (centerline) related to distance $x$ (- - - -), predicted by eq. (3). The maximum velocity of air jet in the foliage, predicted by eq. (21) is also shown: peach on the left, (----), vine on the right (-----), with the experimental value $\left({ }^{\circ}\right)$ at distance $x_{e}$. Graphs are sorted top to bottom for increasing air velocity on exit from the sprayer $v_{x o}$.

The diagram of fig. 6 shows the direct comparison of the values of air velocity $v_{x e}$ predicted by the equation (21) and measured. The experimental value is reported as the mean value with standard deviation bars.

As pointed out in the graph, the experimental mean values are similar to predicted ones with a mean relative error of $3.3 \%$ and a maximum relative error of $6.2 \%$. These errors are fairly acceptable, even if measured values of air velocity reveal standard deviations sometimes relevant.

This means that, to determine the mean value of the resistance factor $k \cdot \rho_{l}$ (for a given crop at a certain growth stage) by direct measurement of air velocity on exit from the foliage and subsequent application of equation (25), a repeated sampling of $v_{x e}$ is always need. 


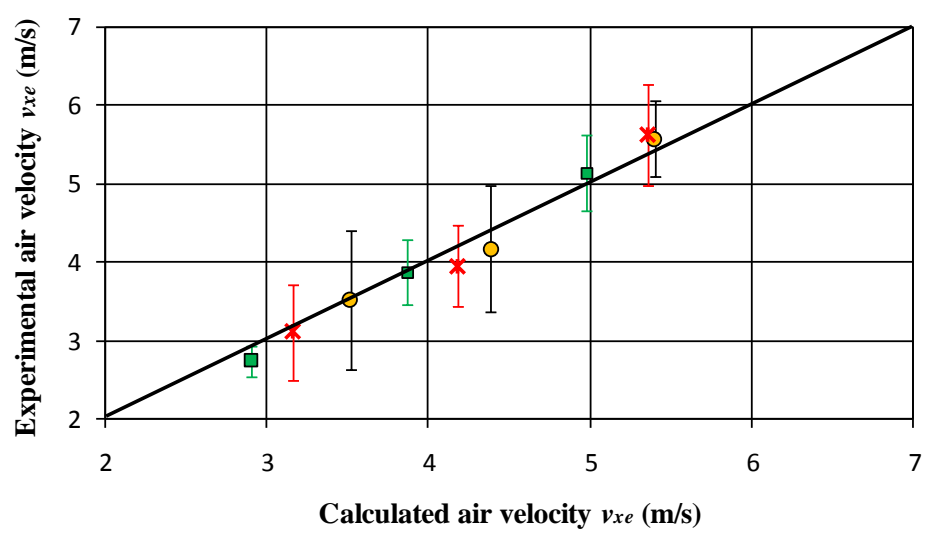

Fig. 6 - Average experimental air jet velocity $v_{x e}$ completed of S.D. vs. calculated air jet velocity by eq. (21): $\square$ apple; * vines; $\circ$ peach.

\section{Conclusions}

Several experimental work confirmed the importance of the velocity of the air jet, produced by the fan of the sprayer, which carries the droplets of plant protection products during spray application in fruit and vine growing. The results agree that excessive air speed increases pesticide losses in the environment, while, on the other hand, when air jet is too slow, uniformity of spray deposition in the canopy gets worse.

For a better understanding of these results and, in view of developing a self-adjustment system of the fan for orchard sprayers, it seemed useful to have an equation to predict the decay of air velocity as a function of the distance from the outlet of the sprayer while air crossing the tree row.

To achieve the equation, the momentum theorem was applied to a control volume inside the foliage, under three assumptions, thus obtaining a differential equation whose integration led to a closed solution (21) easy to implement in a PLC for a future self-adjustment system for orchard sprayers.

The first assumption, related to the disregarding of travel speed $v_{f}$, was legitimate by its tenfold lower value compared to the air velocity $v_{x o}$. The second assumption, about uniformity of leaves distribution in the canopy, that is to say that leaf area density $\rho_{l}$ is constant, was made acceptable by experimentally determining - for each crop and growth stage - of a mean value of the resistance factor $k \cdot \rho_{l}$. This was moreover easy to find, since based on the same integral equation (21), properly rewritten (25). The third assumption, about the inverse proportionality between drag coefficient $c_{r}$ and air velocity $v_{x}$, was verified by a lab test in wind tunnel, which confirmed it for $v_{x} \geq 3$. Finally, the integral equation obtained (21) was submitted to on-field verification on three crops (vine, peach and apple). Results were encouraging with a relative mean error of $3.3 \%$ and maximum $6.2 \%$. Anyway, the variation of experimental measurements of air velocity $v_{x e}$ at the exit of the foliage, as confirmed by standard deviations, resulted wide, particularly on 
peach because of the non uniformity of the canopy of this crop. Therefore, to apply the equation (21) accurately, the preliminary assessment of the mean value of resistance factor $k \cdot \rho_{l}$ for a given crop and growth stage with the equation (25), always requires a repeated sampling of the air velocity out of the foliage.

In conclusion, it is necessary to highlight that the equation (21) proposed for the decay of maximum axial velocity $v_{x}$ of the air crossing the foliage is valid when the $v_{x} \geq 3$, that is to say the range where the integration of the differential equation (17) was possible assuming the parameter $k$ being constant. In the future, it will be interesting to try to extend the integration of the (17) in the range $v_{x}<3$, namely where $k$ is variable (fig. 3).

\section{References}

[1] P. Marucco, M. Tamagnone, P. Balsari, Study of Air Velocity Adjustment to Maximise Spray Deposition in Peach Orchards. The CIGR Ejournal. Manuscript ALNARP 08 009. Vol. X. May, 2008.

[2] F. Pezzi, V. Rondelli, The Performance of an Air-assisted Sprayer operating Vines. Journal of Agricultural Engineering Research, 76 (2000), 331-340. http://dx.doi.org/10.1006/jaer.2000.0540

[3] L. Trefan, Possibilità di valutare la distribuzione di prodotti antiparassitari in frutticoltura (Assessment of agrochemical distribution in orchard). Macchine Motori Agricoli, 5 (1985), 17-25.

[4] R. Holownicki, G. Doruchowski, A. Godyn, W. Swiechowski, Variation of spray deposit and loss with air-jet directions applied in orchards. Journal of Agricultural Engineering Research, 77 (2) (2000), 129-136.

[5] R. D. Brazee, R. D. Fox, D. L. Reichard, F. R. Hall, Turbolent jet theory applied to air sprayer. Transaction of ASABE, 24 (2) (1981), 266-272. http://dx.doi.org/10.13031/2013.34237

[6] D. Friso, A mathematical solution for food thermal process design. Applied Mathematical Sciences, 9 (2015) no. 6, 255-270.

http://dx.doi.org/10.12988/ams.2015.411980

[7] D. Friso, Brake Thermal Efficiency and BSFC of Diesel Engines: Mathematical Modeling and Comparison between Diesel Oil and Biodiesel Fueling. Applied Mathematical Sciences, 8, no. 130 (2014), 6515 - 6528.

http://dx.doi.org/10.12988/ams.2014.46444

[8] S. Pascuzzi, E. Cerruto, Spray deposition in "tendone" vineyards when using a pneumatic electrostatic sprayer. Crop Protection 68 (2015), 1-11. 
http://dx.doi.org/10.1016/j.cropro.2014.11.006

[9] A. I. Fraser, Wind tunnel studies of the forces acting on the crowns of the small trees. Report of the Forest Research, 1962, London.

[10] C. Tsatsarelis, Amortissement interne-externe des rameaux des arbres. Rivista di Ingegneria Agraria, 2 (1979), 17-25.

[11] H. Schlighting, Boundary layer theory, Mc Graw-Hill, New York, 1960.

[12] R. D. Fox, R. D. Brazee, D. L. Reichard, F. R. Hall, Power in an air sprayer jet. Transaction of ASABE, 25 (5) (1982), 1181-1184.

http://dx.doi.org/10.13031/2013.33693

[13] J. M. Randall, The relationship between air volume and pressure spray distribution in fruit trees. Journal of Agricultural Engineering Research, 16 (1) (1971), 1-31. http://dx.doi.org/10.1016/s0021-8634(71)80002-1

[14] A. M. Siddiqui, A. A. Farooq, T. Haroonand B. S. Babcock, A variant of the classical Von Karman flow for a Jeffrey fluid. Applied Mathematical Sciences, 7 (2013), no. 20, $983-991$.

[15] P. H. Gunawan, Numerical study of staggered scheme for viscous Saint-venant equations. Applied Mathematical Sciences, 8 (2014), no. 108, 5349-5359. http://dx.doi.org/10.12988/ams.2014.47580

Received: January 29, 2015; Published: February 19, 2015 